\title{
Síndrome de Susac: reporte de caso
}

\author{
Susac Syndrome: Case Report
}

Sara Arias ${ }^{1}$, Mariana Gómez ${ }^{1}$ y Luis Gustavo Celis ${ }^{1}$

\section{Resumen}

Objetivo. Se describe un caso de una paciente femenina de 23 ańos de edad, con cefalea temporal unilateral izquierda de tipo opresiva, asociado a ataxia, desorientada en espacio y tiempo, y bradipsiquia. Métodos. El abordaje inicial fue hacia una posible esclerosis múltiple y asociada a la clínica y paraclínicos se sospechó una encefalomielitis diseminada aguda para lo cual inician pulsos de metilprednisolona, y dan egreso. Al mes, consulta nuevamente por exacerbación de la sintomatología neurológica dada por pérdida de la fuerza en miembros superiores e inferiores, disartria, relajación de esfínteres, somnolencia, se da como diagnóstico diferencial enfermedad de Devic, y al no obtener la respuesta adecuada a los corticoides se decide manejo con plasmaféresis, además de solicitar exámenes para descartar la patología en cuestión. Resultados. Se enfocó a la paciente con un posible diagnóstico de Síndrome de Susac solicitándose una arteriografía retiniana, y potenciales auditivos evocados.

Palabras clave: esclerosis múltiple, enfermedad de Devic, síndrome de Susac, encefalomielitis aguda, ataxia, hiporeflexia.

\section{Abstract \\ Objective. To describe the case of a 23 year-old female patient disoriented in space and time, with an oppressive left-sided temporal headache, associated with ataxia, and bradypsychia. Methods. A possible multiple sclerosis associated to the clinical and paraclinical tests was suspected at the beginning. An acute disseminated encephalomyelitis was suspected and treated with methylpred- nisolone pulses and patient was discharged. Within a month, she required again due to exacerba- tion of neurological symptoms given by a loss of strength in upper and lower limbs, dysarthria, wetting, and drowsiness. Devic's disease was diagnosed initially. Because response to corticos- teroids was not the expected, it was decided to treat with plasmapheresis; in addition, new tests were requested to rule out the suspected disease. Results. Treatment was focused on a possible diagnosis of Susac's syndrome with an arteriography retinal test and potential auditory evoked.}

Keywords: acute encephalomyelitis, alopsiquia, ataxia, Devic's disease, hyporeflexia, multiple sclerosis, Susac's syndrome

Facultad de Medicina de la Universidad de La Sabana. Campus del Puente del Común, Km. 7, Autopista Norte de Bogotá. Chía, Cundinamarca, Colombia.

Correspondencia: marianagolo@unisabana.edu.co, saraarvi@unisabana.edu.co, luis.celis@unisabana.edu.co

Recibido: 19/02/2015 Aceptado: 15/05/2012 


\section{Introducción}

El síndrome de Susac fue descrito por primera vez por John Susac en 1976 (1) y desde entonces ha sido llamado con diferentes nombres, entre ellos RED-M (retinopatía, encefalopatía, sordera asociada con microangiopatía), síndrome de SICRET (infartos pequeños en tejido coclear, retina y encefálico) (2) y vasculopatía retinococlear. Este síndrome es raro y generalmente muestra predominio del género femenino con una proporción de 3 a 1 . Así mismo, son muy pocos los casos documentados y menos de 100 casos de síndrome de Susac que se han reportado en la literatura (3).

Este síndrome no se diagnostica a menudo, ya que debe ser diferenciado de una serie de enfermedades como la esclerosis múltiple, la encefalomielitis diseminada, lupus eritematoso sistémico, enfermedad de Meniere, migraña, accidentes cerebrovasculares tromboembólicos y enfermedad de Devic (4), entre otros. Por otra parte, muchos pacientes no presentan inicialmente la tríada clínica y muestran formas incompletas de la enfermedad en la que se observa sólo obstrucción de la arteria retiniana sucursal o pérdida de la audición en ausencia de encefalopatía (5).

\section{Reporte de caso}

Con previo consentimiento informado se tiene el caso de una mujer de 23 años de edad, sin antecedentes definidos tanto personales como familiares; quien asiste al Hospital Universitario de La Samaritana (HUS) el día 05/08/2013 por presentar cuadro clínico de 5 días de evolución consistente en cefalea temporal de tipo opresivo de intensidad $8 / 10$, asociado a ataxia, marcha lateralizada, amnesia y bradipsiquia. Una familiar refiere que es la primera vez que cursa con esta sintomatología. La paciente llega con paraclínicos extrainstitucionales de Resonancia Magnética Nuclear (RMN) de cerebro donde se evidencian múltiples lesiones focales puntiformes en ambos hemisferios cerebrales y cerebelosos. Estos podrían corresponder a placas de desmielinización con signos de actividad.
A la revisión por sistemas, ningún dato relevante. Los hallazgos positivos que se encuentran al examen físico son: en la parte neurológica una paciente alerta, con alteración alopsiquica, marcha lateralizada, atáxica, en cuanto a las extremidades simétricas, con sensibilidad conservada, con fuerza 3/5 en miembros inferiores y $5 / 5$ en miembros superiores e hiporreflexia generalizada $(+/++++)$

A su llegada al HUS, es valorada por el servicio de medicina interna, quienes por la sintomatología de la paciente y hallazgos en la resonancia magnética sospechan de Esclerosis Múltiple, solicitan entonces hemograma, potenciales evocados somatosensoriales, visuales y auditivos de tallo cerebral, nueva resonancia magnética con gadolinio, pruebas de función hepática, pruebas de función renal, electrolitos, y VDRL, además de valoración por neurología.

Los paraclínicos de ingreso mostraron unos leucocitos de 9.020, neutrófilos $66.9 \%$, linfocitos $25.4 \%$, hemoglobina $14.4 \mathrm{~g} / \mathrm{dl}$ hematocrito $43.3 \%$ y plaquetas 230.000. VDRL No reactivo. No se evidenció alteración renal, hematológica o hidroelectrolítica.

Tabla Na 1. Resultados LCR en la paciente en dos tomas diferentes.

\begin{tabular}{|c|c|c|c|}
\hline LCR & 09/08/13 & $13 / 08 / 13$ & $\begin{array}{c}\text { Valores } \\
\text { normales }\end{array}$ \\
\hline Color & Incoloro & Incoloro & Incoloro \\
\hline leucocitos & $1 \mathrm{~mm} 3$ & $1 \mathrm{~mm} 3$ & $<5 \mathrm{~mm} 3$ \\
\hline eritrocitos & $144 \mathrm{~mm} 3$ & $423 \mathrm{~mm} 3$ & 0 \\
\hline glucosa & $81 \mathrm{mg} / \mathrm{dl}$ & $72 \mathrm{mg} / \mathrm{dl}$ & $90-$ \\
\hline proteínas & $176,83 \mathrm{mg} / \mathrm{dl}$ & $144,7 \mathrm{mg} / \mathrm{dl}$ & $\begin{array}{c}15,00-- \\
45,00\end{array}$ \\
\hline $\begin{array}{l}\text { Otros } \\
\text { (KOH, } \\
\text { VDRL, } \\
\text { BK,Gram) }\end{array}$ & negativo & Negativo & negativo \\
\hline
\end{tabular}


$\mathrm{Al}$ tercer día del ingreso, se pregunta de nuevo a un familiar quien refiere haber presentado cuadro gripal severo que se dio 10 días antes del inicio de los síntomas actuales. Por el inicio agudo, la presencia de cefalea y alteraciones cognoscitivas asociadas a lesiones desmielinizantes en la sustancia blanca, se piensa en la presencia de Encefalomielitis Aguda Diseminada (ADEM) por lo cual se decide iniciar metilprednisolona 1g/día por 3 días, así como realización de punción lumbar, para definir diagnóstico adecuado.

Hasta entonces, la paciente persistía con el cuadro clínico de debilidad muscular, desorientación temporo-espacial, ataxia por lo que se solicitan estudios para diagnósticos diferenciales: esclerosis múltiple y neuritis óptica, se solicitan bandas oligoclonales y anticuerpos antineuromielitis óptica en suero. La punción lumbar da como resultado un líquido cefalorraquídeo (LCR): encontrando hiperproteinorraquia sin consumo de glucosa, con presencia de eritrocitos, por posible punción traumática, no hay presencia de bacterias en los cultivos, BK negativo, $\mathrm{KOH}$ negativo, Tabla 1. Por evolución favorable de su estado neurológico, se da salida el día 14/08/13 con orden de RMN cerebral con gadolinio extrainstitucional y control en 15 días por neurología para seguimiento de su patología.

Por exacerbación de la sintomatología la paciente consulta nuevamente el día 09/09/13, la acompañante familiar refiere aumento de la debilidad muscular, disartria, estado de somnolencia, relajación de esfínteres en 2 ocasiones, y estreñimiento de 1 mes de evolución. Como hallazgos en el examen físico se encuentra paciente somnolienta, fuerza en miembros inferiores $0 / 5$, fuerza en miembros superiores $3 / 5$, sin signos de irritación meníngea, comprende pero no nomina ni repite, se encuentra hiperreflexica $(+++/++++)$, y con respuesta plantar extensora bilateral.

La paciente presenta un RMN Cerebral Simple con gadolinio que mostró múltiples focos hiperdensos que comprometían sustancia blanca de la corona radiata e igualmente la región gangliobasal a nivel del brazo posterior de la cápsula interna del lado derecho, Figura 1. Estos hallazgos fueron compatibles con esclerosis múltiple por lo que se indica por el servicio de neurología iniciar pulsos de metilprednisolona $550 \mathrm{mg} /$ día por 3 días con previa desparasitación con ivermectina 55 gotas.

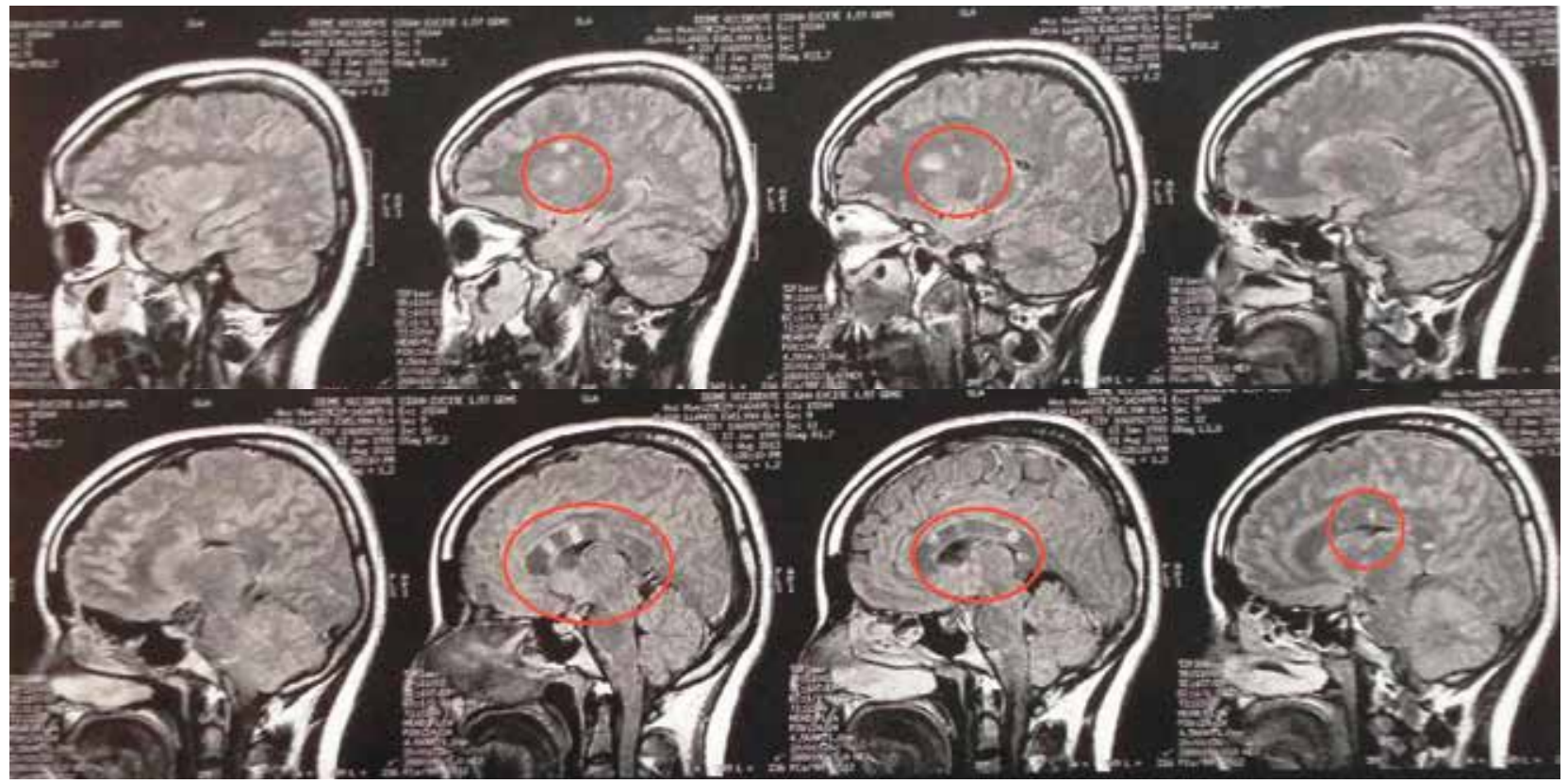

Figura 1. Resonancia Magnética Nuclear de la paciente en la que se muestran múltiples focos hiperdensos que comprometen la sustancia blanca a nivel de la corona radiata y la región gangliobasal. 
Se solicitan nuevos paraclínicos donde se reporta: leucocitos 4990, neutrófilos 56\%, linfocitos 35\%, hemoglobina 16, hematocrito $50 \%$, plaquetas 170000, BUN: 10, creatinina: 0,78, glucosa: 91 $\mathrm{mg} / \mathrm{dl}$, sodio: $138 \mathrm{meq} / \mathrm{l}$, potasio: $4 \mathrm{meq} / \mathrm{l}$, PCR: $5,8 \mathrm{mg} / \mathrm{l}$.

Al cuarto día de reingreso al HUS, la paciente presenta una evolución clínica estable y se solicita una nueva punción lumbar donde se encuentra líquido claro con hiperproteinorraquia $(144,7 \mathrm{mg} / \mathrm{dl})$, persistencia de los hematíes ( $423 \mathrm{~mm} 3)$, y sin otras alteraciones comparado con el estudio de LCR realizado previamente en la anterior hospitalización, Tabla 1.

Se establece que la paciente aún cursa con episodio de encefalomielitis aguda diseminada, y que la respuesta al tratamiento con corticoide endovenosa no ha sido del todo efectiva para la enfermedad, así mismo se sospecha síndrome de Devic por lo que se considera necesario realización de plasmaféresis en 7 sesiones diarias, siendo las últimas 2 interdiarias y traslado a UCI. Al día siguiente, durante la realización de la primera plasmaféresis, la paciente presenta un episodio de pérdida de conciencia asociado a hipotensión y bradicardia, por lo que se decide paso de catéter venoso central y administrar dopamina, paso de un bolo de DAD $10 \%$ y se inicia hidratación, recuperando tensión arterial, frecuencia cardiaca y estado de conciencia, se decide continuar con la plasmaféresis como terapia bajo observación ante posibles complicaciones. Después de este episodio, la paciente presenta una evolución favorable y, posterior a la realización de las sesiones, mejoría del estado clínico, encontrándose alerta, con mejor conexión con el examinador y respondiendo a órdenes sencillas.

Hasta el momento de su estancia en UCI se obtienen resultados de bandas oligoclonales y acuaporina negativo y RMN cervical sin alteraciones por lo que se descarta la sospecha de esclerosis múltiple, así como de Síndrome de Devic (4). Se solicita valoración por oftalmología por nueva sospecha diagnóstica de Síndrome de Susac quienes reportan en el examen de fondo de ojo: microangiopatía e isquemia en capas de fibras nerviosas retinianas, y solicitan angiografía retiniana, potenciales auditivos y electroencefalograma.

El día 11/10/13 la paciente presenta un episodio consistente en movimientos oculares repetitivos y alteración en el estado de conciencia, al examen físico TA: 99/58 Fc: 95 SaO2: 89\%, GLASGOW 10/15 AO4 RV1 RM 5, pupilas midriáticas, hiporreactivas, con nistagmus horizontal no agotable, asociado a sialorrea, y a relajación esfínteres, mayor a 10 minutos de duración, se considera status epiléptico no convulsivo, se considera regular crisis con midazolam, y se inicia impregnación con fenitoina (15 mg/kg/dosis), para luego optimizar manejo farmacológico con levetiracetam $1 \mathrm{~g}$ cada 8 horas e infusión de midazolam $1 \mathrm{mg} /$ hora; posterior a eso la paciente presenta nuevo episodio de deterioro neurológico dado por disminución de la fuerza en extremidades de predominio inferiores $1 / 5$, hiperreflexia generalizada $+++/++++$ y persistencia de la respuesta plantar extensora bilateral. El electroencefalograma (EEG) muestra ondas lentas por periodo post ictal, y los potenciales evocados auditivos están relacionados con un compromiso desmielinizante de la vía auditiva aferente a nivel del tallo o un defecto coclear que explica la pérdi$\mathrm{da}$ a la percepción de frecuencias bajas y medianas.

Se presenta el caso de la paciente a junta médica del HUS, quienes consideran que el cuadro clínico de la paciente se relaciona con un Síndrome de Susac, y deciden realizarle seguimiento en una unidad de cuidado crónico para la rehabilitación física e integral de la paciente.

\section{Discusión}

El Síndrome de Susac es una enfermedad que se caracteriza por presentar una triada clínica clásica (6): encefalopatía, hipoacusia y oclusión de las ramas retinianas arteriales y suele afectar a mujeres jóvenes con edad media de presentación de 30 anos. 
Fue descrita por primer vez en 1979 (1) por Susac, en dos mujeres que sufrían de esta tríada mencionada anteriormente; desde entonces se ha llamado de distintas maneras RED-M (retinopathy, encefalopathy, deafness associated with microangiopathy), SICRET (small infarction of cochlear, retinal and encephalitic tissue) o vasculopatía retinococlear $(7,2)$.

El curso de la enfermedad es propio de cada paciente. La patogénesis de este síndrome se cree está relacionada con reacciones inmunológicas desencadenadas hacia vasos de pequeño calibre, produciendo inflamación y microinfartos en la retina, cerebro y en la región apical de la cóclea (5). A pesar de que la etiología no es conocida, se han realizado estudios de autopsias, en los que se sugiere que el síndrome podría estar ligado a una endoteliopatia $(6,8)$, alteraciones en la coagulación, y microembolización, e incluso se considera su similar origen embriológico, pero realmente ninguna ha sido comprobada.

Desde que se hizo la descripción inicial de este síndrome, menos de 100 pacientes han sido diagnosticadas (3), al considerarse una "enfermedad rara" ha sido subestimada y muchas veces tiende a confundirse con distintas enfermedades para llegar a un diagnóstico, como en la revisión de este caso, muchos pacientes reciben otros diagnósticos y tratamientos consecuentes con los diagnósticos diferenciales, como enfermedad de Devic o neuromielitis óptica $(4,9)$, esclerosis múltiple, migraña, lupus eritematoso sistémico, enfermedad de Meniere, enfermedad cerebrovascular de origen cardioembólico e incluso esquizofrenia (10).

A pesar de considerarse como característica la triada clínica, se han reportado varios casos en la literatura, los cuales para el diagnóstico del síndrome no presentan la triada específica considerándose como incompletos (11-12). En este caso la mujer cumple con criterios de la triada como lo son la encefalopatía dado por la cefalea temporal unilateral izquierda de tipo opresiva, de inicio del cuadro clínico, trastornos cognoscitivos como su pérdida de memoria temporo-espacial, alteraciones en el comportamiento, su bradipsiquia, confusión, y bradilalia. La alteración motora como la ataxia, marcha lateralizada e hiperreflexia generalizada (10), incluso se pudo observar alteración psiquiátrica como labilidad emocional cuando se tocaban temas como el de su hijo, y su mutismo seleccionado.

En cuanto a otro componente de la triada, los trastornos vestibulococleares, en la paciente se documenta principalmente la pérdida de audición una vez se realizan los potenciales evocados relacionados con la dificultad para captar las frecuencias bajas y medias, relacionados con el compromiso desmielinizante versus defecto coclear, en otros casos suele asociarse a otros síntomas como tinnitus, vértigo, náuseas, nistagmus y alteración en el equilibrio $(1,3,5,10)$, la paciente del reporte presentó también alteración en la bipedestación, y aumento del polígono de sustentación.

En cuanto al componente ocular de la triada en el examen de fondo de ojo de la paciente se obtuvo un hallazgo de microangiopatía e isquemia de fibras nerviosas correspondiente al compromiso por la oclusión arterial que genera este síndrome; en algunos reportes también señalan hallazgos de un vaso fantasma, manchas blancas de tipo algodonosas, leves hemorragias intrarretinianas y estrechez arteriolar generalizada, o cuando la enfermedad es sintomática, se presenta como alteraciones en el campo de visión, visión borrosa o ceguera $(1,3,5,7)$.

Por otra parte, el compromiso cerebral puede ser demostrado mediante varios exámenes como RMN, estudio del LCR, EEG, y angiografía cerebral en los que podemos encontrar: en una T2-RM con gadolinio en la cual se encontrarían hallazgos de pequeñas hiperintensidades multifocales en la sustancia blanca y gris del cerebro y el cerebelo, como las de la paciente, mostrados en la Figura 1. $(11,13)$. 
En el examen del LCR se revela un contenido elevado de proteínas y pleocitosis leve, que concuerda con lo que se conoce en la literatura (14); por EEG que puede mostrar un enlentecimiento difuso, e incluso por biopsias cerebrales que muestran pequeños infartos, y en ocasiones por angiografía cerebral demostrando vasos pequeńos que se encuentran ocluidos o estenosados (2).

En conclusión, el Síndrome de Susac, al no existir muchos casos documentados y al no ser una enfermedad comúnmente diagnosticada, no se ha propuesto escalas de diagnóstico claras o tratamiento definido. Entre algunos de los tratamientos coadyuvantes para retraso de morbi-mortalidad se ha hablado de plasmaferesis, también nimodipino, el cual aumenta el flujo sanguíneo cerebral e inmunoglobulinas intravenosas. Hasta ahora el nivel de mayor evidencia clínica se ha demostrado con el uso de corticoides sistémicos $\mathrm{y}$ agentes inmunosupresores (14-16).

El pronóstico de este síndrome parece ser favorable, pero a la vez hay que entender que el curso clínico de la enfermedad se caracteriza por tener ataques recurrentes de uno o más componentes de la triada. La mayoría de las pacientes han retornado hacia una vida normal; sin embargo en algunas personas pueden presentar secuelas de la enfermedad, así como en otras personas este mismo se comporta de manera autolimitada sin presencia de recaídas durante 2-4 años (14).

Actualmente, son pocos casos existentes en el mundo, y aun así no se encuentran reportes de casos en Colombia, siendo este uno de los primeros, por lo cual se considera la importancia de desarrollar guías para facilitar el diagnostico de muchos pacientes que han quedado sin esperanza por múltiples enfoques incorrectos.

\section{Referencias}

1. Susac JO, Hardman JM, Selhorst JB. Microangiopathy of the brain and retina. Neurology 2003; 29:313-316.

2. Schwitter J, Agosti R, Ott P, Kalman A, Waespe W. Small infarctions of cochlear, retinal, and encephalic tissue in young women. Stroke. 1992;23:903-907.

3. Tai Hung Do, Christiane Fisch, and François Evoy. Susac Syndrome: Report of Four Cases and Review of the Literature. AJNR 2004; 25: 382-388.

4. Álvarez Pinzón Andrés Mauricio, Neuromielitis óptica. Revista Salud Bosque 2012;2:35-45.

5. Han Joo Cho, Chul Gu Kim, Sung Won Cho, and Jong Woo Kim. A Case of Susac Syndrome. Korean J Ophthalmol. 2013; 27: 381-383.

6. Magro, Cynthia MMD; Poe, Jonathan C, PhD; Susac, John O,MD.American. Susac Syndrome: An Organ-Specific Autoimmune Endotheliopathy Syndrome Associated With AntiEndothelial Cel Antibodie. Journal of Clinical Pathology 136.6 (Dec 2011): 903-12.

7. Department of Ophthalmology Luton and Dunstable NHS Trust Luton, UK. V Sandhya and N Anand. Susac Syndrome. Eye 2002;16, 788-790.

8. McLeod DS, Ying HS, McLeod CA. Retinal and optic nerve head pathology in Susac's syndrome. Ophthalmology. 2011 March; 118: 548-552.

9. Thomas C, Mini S, Thomas SV. Rescue plasmapheresis in a cas of severe steroid unresponsive devic's nuromyelitis optica. Ann Indian Acad Neurol 2006; 9:42-45.

10. Lammouchi, Turkia M. Bouker, Samira M. Grira, Mounir T. Benammou, Sofien A. Susac's syndrome. Saudi Med J 2004;25: 222-224.

11. Bienfang, Don C/DC; McKenna, Michael J/MJ; Case records of the Massachusetts General Hospital. Case 24-2011. A 36-year-old man with headache, memory loss, and confusion. N Engl J Med 2011; 365:549-559.

12. Prakash, Gunjan; Jain, Shephali; Nathi, Tirupat. Susac's syndrome: First from India and youngest in the world. Indian Journal of Ophthalmology 61.12 (Dec 2013): 772-773.

13. Kleffner I, Duning T, Lohmann H, et al. A brief review of Susac syndrome. J Neurol Sci 2012;322:35-40.

14. Ortiz Alberto, Roverano Susana, Paira Sergio. Síndrome Susac: presentación de un caso y revisión de la literatura. Revista Argentina de Reumatología 2010;21:29-32.

15. Dörr J, Krautwald S, Wildemann B, et al. Characteristics of Susac syndrome: a review of all reported cases. Nat Rev Neurol 2013; 9:307-316.

16. T.A. Hardy, R.J. Garsia, G.M. Halmagyi, S.J. Lewis, B. Harrisberg, M.J. Fulham, et al. Tumour necrosis factor (TNF) inhibitor therapy in Susac's síndrome. J. Neurol. Sci., 302 (2011), pp. 126-128. 